\title{
Medium, Performanz, Inhalt: Eine theoretische Metastudie zum mediengestützten Lernen
}

\author{
Medium, performance, and content: \\ A theoretical meta-analysis on media-based \\ learning
}

\begin{abstract}
Media-based learning does not necessarily make sense nor is it necessarily effective, for that matter, simply because it uses a medium. Rather, there are several variables that can and do influence its success: the very medium, the content, and the learner's cognitive styles. All variables have to be taken into consideration, alone and interacting, in order to decide whether and where media-based learning is to be used, and where it might be counterproductive.
\end{abstract}

KEYWORDS: media-based learning, medium, performance, learning styles, content.

\section{IST MEDIENGESTÜTZTES LERNEN SINNVOLL?}

In der Frühphase mediengestützten Lernens hat Clark 1983 in einem damals viel zitierten Aufsatz angedeutet, dass das Medium vermutlich keine entscheidende Konsequenz für den Lernprozess habe. Im Rahmen eines von mir verantworteten Drittmittelprojekts, das Chancen und Probleme eines mediengestütztes Sprachunterrichts untersuchen sollte (Projekt "Sprachnetzwerke“ im Modelversuchsprogramm „Lebenslanges Lernen“ der „Bund-Länder-Kommission für Bildungsplanung und Forschungsförderung", Förderkennzeichen: A6682SL 01), wurde aber schnell und bereits im Rahmen der Literatursichtung im Vorfeld deutlich, dass eine Bilanz des mediengestützte Lernens komplexer (auch: nachweisbar) ist, als gemeinhin vermutet (Giessen 2004). 
Zunächst (und sehr allgemein) konnte zwar bestätigt werden, dass die Mehrzahl der Studien eine gewisse Gleichwertigkeit des traditionellen und des mediengestützten Lernens erkennen lassen. Da es sich um quantitative Studien handelte, wurden in der Regel Schulklassen oder Lernergruppen miteinander verglichen. Dabei war die soziographische Zusammensetzung der Gruppen, aber auch der Lerninhalt möglichst identisch, so dass die einzige veränderte Variable im mediengestützten Unterricht lag. Mediengestützter Unterricht hat sich im Rahmen dieser experimenteller Studien, also im direkten Vergleich mit traditionellem Präsenzunterricht, im Schnitt als nicht schlechter erwiesen als die herkömmlichen Unterrichtsmethoden allerdings in der Regel auch nicht als signifikant besser.

Allerdings gab es Beobachtungen, die daraufhindeuteten, dass einzelne Schüler mit mediengestütztem Lernen besser zurechtkommen als mit traditionellem Unterricht, während andere Schüler umgekehrt mit mediengestütztem Lernen größere Probleme hatten, weniger gut zurecht kamen und sogar schlechter abschnitten, als dies im traditionellen Unterricht der Fall gewesen wäre. Demnach könnten die experimentellen Studien also eine Zweiteilung der Schülerschaft in diejenigen, die vom Lernen mit dem Computer profitieren, und andere, die weniger gut mit mediengestütztem Unterricht zurechtkamen, verstecken.

Welche weitere Variable könnte dafür eine Rolle spielen? Wallace und Mutooni schildern bereits 1997, dass die Nutzer eines computergestützten Angebots im Gegensatz zu den Teilnehmern einer Präsenzveranstaltungen die Tendenz zeigten, so lange bei den einzelnen Themen zu verweilen, bis ein hohes Verständnis des Lernstoffs erreicht war. Sie lernten auch flexibler über den ganzen Tag verteilt als ihre ,Konkurrenten'. Offensichtlich benötigt das mediengestützte Lernen also mehr Zeit als der traditionelle Unterricht. Studierende, die diese Zeit nicht aufbringen konnten oder wollten, profitierten nicht von mediengestützten Unterricht, im Gegenteil. Pitman, Gosper und Rich haben 1999 umgekehrt die Noten von 348 Studierenden analysiert und auf das Lernverhalten der Studierenden bezogen; dabei zeigte sich, dass Studierende mit besseren Noten häufiger und regelmäßiger die computergestützt präsentierten Zusatzangebote abgerufen und genutzt hatten, als die Studierenden mit den schlechteren Noten. Schulman und Sims konnten 1999 diese Vermutung ebenfalls bestätigen - sie haben beobachtet, dass die besseren Lerner lieber und häufiger mediengestützt lernten und arbeiteten, während schlechtere Lerner eher traditionelle Lernformen wählten.

Unterschiedliche Zeitvolumina wären demnach ein Kriterium, das für den Erfolg mediengestützten Unterrichts bedeutsam sein kann. Man muss offenbar genug Zeit haben, um mit dem Computer zu lernen - offensichtlich mehr Zeit, als beim ,klassischen Unterricht'; wenn man aber diese Zeit auf- 
bringt oder auch aufbringen will, scheint man auch einen größeren Lernerfolg zu haben. Dies bestätigen weitere Befragungen, qualitative Studien und Evaluationen, beispielsweise Scott, Durnell Cramton, Gauvin, Steinke und Patterson bereits im Jahr 1997, die 123 distant learners im Rahmen eines multinationalen E-Learning-Projekts nach ihren Einschätzung befragt haben. Erneut gaben die Probanden an, dass ihnen große Zeitvolumina und eine angemessene Zeitkoordination ausgesprochen wichtig waren. Ähnlich ist schließlich das Ergebnis von Ward und Newlands aus dem Jahr 1998. Die Versuchspersonen gaben als entscheidende Vorteile an, dass sie größere Freiheiten hatten, den Zeitpunkt und das Tempo des eigenen Lernens zu bestimmen. Dies führte auch hier dazu, dass die Studierenden länger bei den Lerninhalten blieben und dass sie solange studierten, bis sie alle Informationen aufgenommen und verarbeitet hatten. Die Hauptkonsequenz von Ward und Newlands ist deshalb, erneut, dass durch mediengestütztes Lernen mehr Inhalte aufgenommen werden können, dass dazu aber auch mehr Zeit benötigt wird. Schließlich bestätigen beispielsweise auch Usip und Bee 1998 die Bedeutung des Faktors ,Zeit'. Sie untersuchten, welche Merkmale die Nutzer von Online-Angeboten von den Nutzern traditioneller Vorlesungen unterschieden. Auch bei ihnen gab es als wichtigstes Ergebnis, dass die Nutzer computergestützter Angebote zunächst offenbar im Schnitt nicht schlechter oder sogar besser waren als ihre ,Konkurrenten'. Ganz entscheidend war aber auch hier, dass sie mehr Zeit benötigten, um den Inhalt dann aber in der Regel auch intensiver aufzunehmen, beispielsweise Tests und Leistungsüberprüfungen durchzuarbeiten. Erfolgreich war letztlich nur der Teilnehmerkreis, der so viel Zeit auch verfügbar hatte.

Allerdings hat sich im Lauf unseres Projekts gezeigt, dass sich auch hinter diesem Befund eine weitere Erklärungsebene verbergen kann. Offenbar spielt auch die objektiv zur Verfügung stehende Zeit nicht die entscheidende Rolle. Viele Schüler nahmen sich die Zeit nämlich bewusst nicht, weil sie keine Freude am mediengestützten Lernen hatten. Unsere Befragungen haben ergeben, dass dies vor allem an zwei Faktoren hing: den Lerninhalten, sowie am Lernertypus. Natürlich gibt es Formen des mediengestützten Lernens, bei denen, ähnlich zum traditionellen Unterricht, auf einzelne Lerner und deren individuelle Vorlieben und Schwierigkeiten eingegangen werden kann, etwa bei der Videokonferenz (Bufe, Giessen 2005). Teilweise gilt dies auch für andere sogenannte "social media", zumindest (erneut) in Abhängigkeit zur Art und Weise ihres Einsatzes. Dagegen dominiert bei mediengestützte Lerneinheiten, Tutorials, Übungen usw., die ja in der Regel standardisiert sind, das Medium, so dass es hier mitunter lediglich die Entscheidung der Akzeptanz oder der Verweigerung als Handlungsalternative der Lerner existiert. Sie werden ja teilweise gerade deshalb produziert, 
um mit einer gewissen Effizienz Lerninhalte für mehrere (viele) Lerner zur Verfügung zu stellen. Die Folge besteht darin, dass Lerner, für die andere Lernformen besser geeignet wären, gezwungen sind, sich dem medial präsentierten Inhalt zu unterwerfen.

Natürlich gibt es auch mediengestützte Angebote, die nicht von einer Schule beziehungsweise von Lehrern aufgezwungen werden - etwa CDs mit Sprachkursen oder entsprechende Online-Angebote. Hier ist natürlich kein direkter empirischer Vergleich zwischen zwei Lernergruppen möglich. Grundsätzlich scheinen solche Angebote ja auf eine gewisse Nachfrage zu stoßen; dies könnte ein Indikator für ihre Wirksamkeit sein. Auch hier ist aber fraglich, ob es nicht im Vorfeld eine Selektion der potentiellen Lerner gibt - ob eben nur diejenigen solche Angebote erwerben, die gut und zielführend mit mediengestütztem Lernmaterial umgehen können. Andere kaufen solche Angebote womöglich gar nicht erst. Zudem hat sich schon relativ früh gezeigt, dass auch die Motivation zur Nutzung mediengestützter Lernangebote differenziert zu sehen ist; zumindest ist die Qualität des Lernens oder das Ergebnis nur eine und mitunter noch nicht einmal die primäre Kategorie bei der Entscheidung für ein solches Produkt - andere Kategorien von genauso großer, teilweise gar von größerer Bedeutung sind beispielsweise die nun mögliche räumliche und zeitliche Flexibilität beim Lernen und die so erreichbaren Mehrwerte. Erwartungen an die pädagogische Qualität und die Aufwand-Ergebnis-Relation treten dagegen zurück (Kariya 2003). Der Markt für Bildungsmedien ist also ebenfalls kein Argument gegen die Vermutung, dass es unterschiedliche Lernertypen gebe, von denen manche auch Schwierigkeiten mit medialem Lernen haben.

Dass es unterschiedliche Lernertypen gibt, ist im Übrigen schon lange bekannt; es sei auf die historische Studie von Kurt Lewin aus dem Jahr 1942 verwiesen. Dass es unterschiedliche Typen beim Umgang mit dem Computer gibt, ist ebenfalls kein neue Erkenntnis; populär wurde diese Einsicht beispielsweise durch das Buch von Sherry Turkle bereits im Jahr 1984. Obwohl mithin die Existenz unterschiedlicher Lernertypen auch im Kontext mediengestützten Lernens nicht überraschen sollte, ist auffallend, dass sie nur selten berücksichtigt werden. Der Grund liegt, wie bereits erwähnt, vermutlich vor allem darin, dass mediengestützte Lerneinheiten in der Regel für Gruppen oder Klassen vorbereitet und organisiert beziehungsweise als Materialien für Nutzergruppen (und eben nicht für individuelle Lerner) produziert werden, so dass auch die Evaluation auf Gruppenebene erfolgt. Wenn nun eine solche Evaluation ,im Schnitt' keine schlechteren Ergebnisse als beim traditionellen Unterricht zeitigt, fällt die Missachtung individueller Lernertypen (zumindest im Kontext der Evaluation) kaum auf. So kann es sein, dass in der Tat verschiedene Lerner durch mediengestützte Lernange- 
bote (wenngleich vielleicht zum Preis eines erhöhten Zeitaufwands) profitieren, während andere Lerner, die größere Schwierigkeiten haben, mit ihren Problemen in den Evaluationen ,untergehen'. Die Lernersicht spielt, da es sich um eine individuelle Kategorie handelt, die quantitativ schlechter darstellbar ist, für die Bewilligung vom Mittel zur Produktion von Lerninhalten oder für Rückmeldungen von Aufsichtsbehörden bislang noch kaum eine Rolle. Aus diesem Grund gibt es Studien, die sich mit Lernertypen im Kontext des mediengestützten Lernens befassen, noch nicht sehr lange. Die Einsicht, dass die Berücksichtigung der ,Lernersicht' (Ehlers 2004) beziehungsweise individueller, kognitiver Lernstile für mediengestütztes Lernen von Bedeutung ist, hat erst seit kurz vor Mitte des vergangenen Jahrzehnts zu entsprechenden Studien geführt. In jedem Fall ist deutlich, dass die Vorstellung, es existiere eine Gleichwertigkeit des mediengestützten und des traditionellen Unterrichts, wichtige Aspekte ignoriert - übrigens bezogen auf beide Lernformen: Manche Lerner profitieren von mediengestütztem Lernen offenbar auch deutlich stärker als vom traditionellen Unterricht (Giessen 2004: 44-47). Was also sind die Variablen, die mediengestütztes Lernen erfolgreich(er) werden lassen - und wann sollte man vielleicht besser darauf verzichten? Sicher gibt es viele situationale und individuelle Aspekte, die sich im Einzelfall auswirken. Auf einer überindividuellen Ebene spielen offenbar das Medium beziehungsweise die Art der mediengestützten Lernmaterialien und der Lernertyp die entscheidenden Rollen. Auf diese Aspekte beziehungsweise Variablen will ich mich daher im Folgenden konzentrieren.

\section{INHALTE, MEDIEN, LERNERTYPEN}

Grundsätzlich - und losgelöst vom mediengestützten Lernen - ist ein Gemeinplatz, dass nicht alle Inhalte mit allen Medien gleich gut übermittelt werden können. Ein Beispiel soll dies verdeutlichen. Warum sind es zumeist Zeitungen und Zeitschriften, die investigativ berichten und gar Krisen des politischen Systems auslösen, wie die "Washington Post" in den USA die Watergate Affäre? Warum gelingt dies einer Tageszeitung, die von relativ wenigen (wenngleich sicherlich einflussreichen und meinungsbildenden) Menschen gelesen wird, nicht aber dem Fernsehen, das von viel mehr $\mathrm{Zu}-$ schauern gesehen wird? Ein Blick auf die Fernsehberichte jener Zeit verdeutlicht schnell, warum dies so ist. Die Fernsehreporter filmten nämlich die "Washington Post" ab, dazu noch das Watergate Gebäude von außen und selbstverständlich auch das Weiße Haus. Aber sie hatten natürlich keine Bilder dessen, was im Rahmen der Watergate Affäre geschehen ist. Investigativer Journalismus in Bezug auf personenbezogene Skandale ist 
demnach mit dem Fernsehen kaum machbar, weil dort eben alles bebildert werden muss. - Dies ist natürlich anders bei investigativem Journalismus, der vom Bild lebt. Wenn ein Kamerateam in einem Supermarkt verschimmelte Lebensmittel aufspürt, ergibt das eindrucksvolle Bilder und hat eine starke Wirkung in der Öffentlichkeit. Aber da, wo es um nicht mehr beobachtbare, weil in der Vergangenheit durchgeführte Taten von Personen und um ihr oft heimliches Verhalten geht, gibt es keine entsprechenden Bilder, und da es fast unmöglich ist, ohne authentisches Bildmaterial der handelnden Personen - und ohne die Chance, es zu drehen - aufklärende Reportagen über deren Verhalten zu erstellen, wird es in Fernsehsendungen in der Regel auch gar nicht versucht. Dieser Verzicht auf entsprechende Themen ist Folge der Einsicht in die Notwendigkeiten des Mediums. Dagegen lassen sich solche Reportagen sehr wohl für eine Zeitung erstellen. Hier kann beschrieben werden, wie die Informationen recherchiert worden sind. Wenn deshalb solche Skandale durch Presseberichte öffentlich werden, dann fast immer durch Printmedien. Das Medium entscheidet also - in diesem Beispiel sehr eindrücklich -, welche Inhalte dargestellt werden können und wie sie wirken. Diese Erkenntnis gilt aber offenbar auch für Lernmaterialien. Auch hierfür ein Beispiel: Im Rahmen des Projekts, bei dem es um das Thema ,Sprachenlernen und Medien' ging, wurden Videoclips für ein Multimediaangebot produziert, auf denen Muttersprachlern zu sehen und hören waren (Giessen 2006). Die Integration authentischer Sprachbeispiele schien ein deutlicher Mehrwert des Mediums darzustellen; insbesondere sollte das Verstehen der Sprache durch synchrones Erleben von Mundbewegungen und lautlichen Äußerungen erleichtert werden. Die Integration in ein Multimediaangebot bedeutete, dass das Videomaterial standardmäßig nicht bildfüllend (wie beim Fernsehen) dargestellt wurde, sondern etwas mehr als ein Viertel des Bildschirms einnahm, die Mundbewegungen waren damit sehr gut zu erkennen. Natürlich konnten die Lerner auf eine bildfüllende Darstellung umschalten; dies erforderte aber ein aktives Eingreifen. In der Normaldarstellung war die Frame-Lösung Teil des Multimediaangebots, das weitere Mehrwerte aufzuweisen schien. So waren beispielsweise stets die Navigationsleiste sowie ergänzende Informationen (auch zu den Videoclips) verfügbar. Interessant ist nun, dass die Testnutzer die Videosegmente nicht auf die Art und Weise nutzten, wie wir uns das vorgestellt hatten. Teilweise brachen sie die Anwendung ab, bevor sie zu Ende war. Wie kam es zu diesem unbefriedigenden Verhalten? Wir beobachteten die Nutzer und führten zudem verschiedene Interviews. Als ein Ergebnis konnte festgehalten werden, dass es zunächst nicht an den videobasierten Inhalten selbst lag. In einer Vollbilddarstellung fanden die Probanden die Videoproduktionen ansprechend, interessant und didaktisch hilfreich. Vielmehr haben mediale 
Charakteristika das unbefriedigende Nutzerverhalten verursacht. Ein Hauptproblem beim Betrachten von Videoclips in computergestützten Multimedia- Angeboten scheint die geringe Distanz zum Computer-Monitor zu sein. Die Nutzer sitzen sehr nahe vor dem Monitor, weil sie Icons oder Hyperlinks exakt mit der Maus treffen müssen. Sie fixieren in der Regel gezielt nur einen Bereich des Monitors, oftmals gar nur einen Punkt. Natürlich führt dies zu einer Verengung der Aufmerksamkeit. Des Weiteren ist die Zeichenauflösung von Computermonitoren relativ gering, mit der Folge, dass die Augen rascher ermüden. Schließlich ist die Körperhaltung nahezu unveränderbar. Der Computer-Monitor kann ja nicht so leicht verschoben werden, wenn die Sitzposition unbequem wird. Gerade dann, wenn man längere Zeit auf nur einen Punkt des Monitors blickt, verkrampft sich die Haltung ganz besonders, was die Ermüdungseffekte weiter verstärkt. Alle befragten Studierenden bestätigten daher, dass es sie ,anstrenge', längere Zeit auf einen Punkt zu blicken, auch wenn sich die Informationen auf diesem Punkt bewegen. Dies kann bezüglich der Videoclips in computergestützten Multimedia-Angeboten zu verschiedenen Handlungskonsequenzen führen. Die Nutzer schauen nicht lange auf das relativ kleine Videoframe, sondern lassen die Augen wandern. Dies ist relativ leicht möglich, denn es gibt noch weitere Frames und Informationen, welche die Aufmerksamkeit auf sich ziehen und als zusätzliche Informationen neben dem Videobild beabsichtigt sind, denn sie betten das Bild in eine visuelle Umgebung ein, die vor allem auch eine reiche Informationsumgebung ist. In der Tat haben viele Studierende bestätigt, dass sie einen Clip zwar laufen lassen, aber nicht lange zusehen. Sie haben häufig sogar den subjektiven Eindruck, dass sie dem Inhalt dennoch folgen können, weil sie ja noch die akustischen Informationen hören, und dass sie also nichts versäumen. Aber sie sind zumindest und zwangsläufig weniger konzentriert - und die visuellen Zusatzinformationen entgehen ihnen dann natürlich ganz. - Offenbar besteht eine weitere Handlungskonsequenz häufig darin, die Möglichkeiten der Interaktivität, die es ja erstmals auch beim Bewegtbild (bei Time Based Media) gibt, zu erproben. Viele Nutzer sehen sich den Anfang einer Produktion an und fahren dann mit dem Schieberegler im Verlauf des Films nach vorne, um zu überprüfen, ob es sich lohnt, den Clip insgesamt anzusehen. Wenn sie den Eindruck haben, dass es doch wichtig sein könnte, den gesamten Film zu sehen, suchen sie in der Regel die Stelle, an der sie anfangs ,ausgestiegen' sind. Sie starten also nicht von neuem, denn es wird als langweilig empfunden, eine Passage, die schon bekannt ist, ein zweites Mal anzusehen. Nun ist es aber schwierig, exakt die Stelle zu finden, an der der Film zunächst verlassen wurde. Mit dem Schieberegler kann man nur ungefähr eine Stelle ansteuern, und kaum jemand merkt sich Timecode-Angaben, wenn er mit dem Schieberegler hin- 
und herfährt. In der Regel finden die Nutzer also nicht die exakte Stelle des Ausstiegs aus dem Film, so dass sie entweder doch eine Passage doppelt sehen, oder es fehlt ihnen ein Stück. Dann verlassen Zuschauer häufig die Videosequenz, weil sie den Inhalt nicht rekonstruieren können. Es sei allerdings (erneut) darauf hingewiesen, dass diese Handlungsalternativen natürlich nur bei Videos auftreten, die in eine multimediale Umgebung eingebettet sind, wo also das Videobild relativ klein ist und daneben die Navigationsleiste oder andere Fenster zu sehen sind, die den Blick auf sich lenken. Dort, wo der Computer-Monitor mit einer ,Vollbild-Darstellung' ausschließlich als ,Fernseher-Ersatz' genutzt wird, gilt dies natürlich nicht. Aber dann ändern sich ja auch viele der äußeren Rahmenbedingungen. Die Sitzhaltung ist eine andere - dann ist der Computer nur der Kanal für eine Fernsehproduktion, die auch als solche konsumiert wird. Das beschriebene Verhalten tritt auch dann nicht auf, wenn im Klassenverbund oder im Seminar gearbeitet wird und die Studierenden dazu angeleitet werden, die Informationen jeweils gleichzeitig zu sichten. Die Beschreibung verdeutlicht aber bereits die Unangemessenheit einer solchen Situation. Immerhin ist dann offenbar der soziale Druck, der von den Dozenten ausgeht oder von den Kommilitonen, die sich gegenseitig beobachten, so groß, dass die Nutzer in der Regel die Videoanwendungen zu Ende sehen, ohne einzugreifen. Multimediaangebote sind aber klassische Selbstlernmedien; und in diesen Situationen treten die beobachteten Schwierigkeiten mit den authentischen Videomaterialien fast ausnahmslos auf.

Es scheint also ein Missverständnis zu sein, für computergestützte multimediale Selbstlernangebote ,authentisches Sprachmaterial' produzieren zu wollen, um Studierenden einen leichteren Zugang zur Zielsprache zu ermöglichen. ,Authentische Sprachmaterialien' eignen sich im Zweifelsfall eher für Videokassetten beziehungsweise DVDs - dort können aber die anderen Vorteile einer computergestützten Multimedia-Produktion, die insbesondere für das autonome Lernen von entscheidender Bedeutung sind, nicht genutzt werden. Eine Videokassette oder eine DVD schließt beispielsweise Medienwechsel und damit direkte, ins Medium integrierte Übungsaufgaben aus. Im Kontext computergestützter Multimedia-Techniken sind Videoclips mit authentischen Sprachbeispielen dagegen zumindest uneffektiv, obwohl es zunächst so naheliegend und sinnvoll zu sein scheint, sie einzusetzen. Das unterschiedliche Verhalten beim Betrachten von Videoclips je nachdem, ob sie alleine oder im Gruppenkontext genutzt werden, deutet des Weiteren darauf hin, dass beim mediengestützten Selbstlernen offenbar auch ein besonders hohes Maß an Selbstdisziplin notwendig ist. Der Grad der Selbstdisziplin ist nun aber vor allem individuell geprägt. Das Beispiel des mediengestützten Lernens mit Hilfe ,authentischer Sprachbeispiele auf Video' 
verdeutlicht also gleichzeitig, welch enger Zusammenhang zwischen medialer Produktion, ihren Zwängen (und Möglichkeiten) einerseits, und dem individuellen Lernertypus andererseits existiert. Die Wechselwirkung zwischen mediengestütztem Lehrmaterialien und Lernertyp ist in verschiedenen Zusammenhängen immer wieder aufgefallen. 2005 haben Bufe und Giessen (und analog beispielsweise 2007 Bailey und Iqbal) betont, dass interaktive Online-Kommunikation (die ja eine deutlicher Mehrwert des mediengestützten Sprachenlernens zu sein scheint, da sie mit Muttersprachlern erfolgen kann) in überraschendem Maß darunter leidet, dass tatsächlich Spontaneität in diesem Kontext kaum möglich ist. Auch bei synchronen Kommunikationsszenarien wie beispielsweise der Videokonferenz kommt es zu mehr oder weniger langen und störenden zeitlichen Verzögerungen. Grundsätzlich fehlen auch verschiedene kommunikative Zusatzinformationen, so dass Ironie, Genervtheit oder Langeweile nicht erkannt werden. Dies kann die Kommunikation durchaus beeinträchtigen.

$\mathrm{Zu}$ den Medieneffekten kommen die individuellen Effekte. Dies fängt damit an, dass die Wahrnehmungsfähigkeit höchst unterschiedlich ist (Montgomery 1999). Visuelle Lerner werden am besten bedient, wenn sie grafische Darstellungen erhalten; auditive Lernertypen sollten Informationen eher akustisch aufnehmen (Ferrari, Sternberg 1998). Die Nutzung mediengestützter Lernmaterialien wird also nie gleich sein; für manche Lerner eignen sich spezifische Medien, für andere eher nicht. Es wird also schwer sein, allgemeine Regel zum Medieneinsatz aufzustellen. Workman (2004) konnte sogar zeigen, dass es Qualitätsunterschiede beim Bearbeiten von Aufgaben und mithin beim Lernen in Abhängigkeit davon gibt, ob die Inhalte über das World Wide Web oder eine CD-Rom zur Verfügung gestellt werden (Workman 2004). Das Lernen im WWW war stärker sozial ausgerichtet und hat mehr Interaktionen benötigt und hervorgerufen; vermutlich, so kann hinzugefügt werden, lenken beim $W W W$ die Programme oder auch nur Möglichkeiten im Hintergrund ab. In jedem Fall war das mediengestütztes Lernen über das $W W W$ weniger strukturiert. Dieses Medium erwies sich aber als positiv für soziale Lerner. Dagegen war das Lernen via $C D$-Rom eher monoton, aber deutlich stärker strukturiert; dies war positiv für Lerner, die introspektiv arbeiteten. Es konnte sogar gezeigt werden, dass diejenigen Lerner, die leichter mit abstrakten Inhalten umgehen konnten, besser webbasiert lernten. Dagegen arbeiteten diejenigen Lerner, die konkrete Informationen bevorzugten und diese Schritt für Schritt aufnehmen wollten, liebe mit der autodidaktischen CD-Rom. Es hat sich also gezeigt, dass bereits die Entscheidung wichtig ist, ob computerbasierte Lehrmaterialien für das Web oder den heimischen Computer produziert werden; je nach Lernertyp gab es spezifische Vor- und Nachteile. 
Empirische Beobachtungen der Bedeutung von Medium und Lernertyp reichen bis in die neunziger Jahre zurück (Fussell, Benimoff 1995), und es gibt inzwischen viele ähnliche Beobachtungen (vergleiche etwa Tamim et al. 2011), so dass die eingangs erwähnte Vermutung von Clark (1983), dass das spezifische Medium für den Lernprozess irrelevant sei, heute wohl nicht mehr akzeptiert sein dürfte. Insgesamt kann festgehalten werden, dass es wohl keine ,allein-seligmachende' Methode gibt, medienbasierte Lehrmaterialien $\mathrm{zu}$ erstellen. Offenbar sind viele Ergebnisse individuell, situativ und kontextabhängig (Bangert 2008). Dennoch ist es möglich, Aussagen zu treffen, in welchem Kontext medienbasiertes Lernen (in jeweils spezifischen Medien) sinnvoll und effektiv ist. So wurde bereits verschiedentlich versucht, diese Unterschiedlichen Auswirkungen theoretisch zu fassen.

\section{THEORETISCHE ANMERKUNGEN}

Insbesondere bezüglich des Einsatzes von Medien gibt es bereits verschiedene Theorien. Bekannt sind etwa die Ansätze Herbert Marshall McLuhans (1962) oder die damit verwandte "media richness theory" von Richard Daft und Robert Lengel (1984). Je nachdem, wie sehr die Lerner (oder allgemein: die Nutzer) vom Medium absorbiert werden, sprechen Daft und Lengel von unterschiedlichen Graden der "media richness": Weniger ,reiche' Medien sind nicht intensiv genug, die gesamte Fokussierung zu erreichen. Beispiele sind etwa Diskussionsforen, Chats, E-Mail oder ähnliche Formen. Weitere mediale Mängel, die dazu führen, dass der jeweilige mediale Einsatz ,schwach' wirkt, sind etwa das Fehlen eines unmittelbaren Feedbacks innerhalb des genutzten Mediums. Die Folge sind fast zwangsläufig Aufmerksamkeitsdefizite und Unsicherheit bezüglich zu absolvierender Aufgaben. In der weiteren Folge bedeutet die Nutzung ,schwacher' Medien, dass Lernen (sowohl beim Selbstlernen, noch stärker aber natürlich beim kollaborativen Lernen, vergleiche dazu auch Mason 2004) größere kognitive Anstrengungen erfordert, die bereits damit beginnen, herauszufinden, was überhaupt gewollt ist, und ob dies dem dient, was man selbst will. Dies wäre der Grund, warum im Kontext medialen Lernens mehr Selbstdisziplin notwendig ist als in traditionellen Lernsituationen. - Relativ neu dagegen ist der Versuch, die medientheoretischen Aussagen mit Untersuchungen zum Lernertyp zu koppeln. Diese Überlegungen spielten (allzu) lange keine Rolle (Flavell 1992; Sternberg, Grigorenko 1997), obwohl es grundsätzlich viele verlässliche Aussagen über unterschiedliche kognitiver Lerntypen gab (Grigorenko, Sternberg 1995). Die Studien bezogen sich aber üblicherweise auf den traditionellen Unterricht; dass es dort Unterschiede bei der Informa- 
tionsverarbeitung und beim Lernen gibt, ist eindeutig (Rehder, Hoffmann 2005). Die Verbindung mit dem Medieneinsatz lässt sich aber erst ab den neunziger Jahre beobachten (zum Beispiel Pillay 1998). Entscheidend ist, wie ein Lerner Informationen aufnimmt und verarbeitet (Hayes, Allinson 1998) und in der Folge Probleme löst und Entscheidungen trifft (Gardner 2009). Ein richtungsweisender theoretischer Ansatz dazu ist etwa die "cognitive load theory" (Hazeltine et. al. 2006); verwandte Überlegungen stammen von Robert J. Sternberg (1997). Seine "theory of mental self-government" ist recht komplex und daher mitunter schwer zu operationalisieren. Sie geht davon aus, dass Lerner unter idealen (und freien) Bedingungen den Lernprozess im Einklang mit ihren kognitiven Möglichkeiten organisieren. Dies führe zu unterschiedlichen Formen mentaler Repräsentationen beziehungsweise unterschiedlichen Kodierungen. Unterschiedliche Lerner benötigen daher unterschiedliche Lernsituationen und -strategien. Manche lernen besser im Kontext interaktiver Gruppenprozesse, andere benötigen Ruhe, um sich konzentrieren zu können. Dieses Beispiel wurde deshalb gewählt, weil es naheliegt, es (zunächst theoretisch) mit den genannten Medientheorien zu koppeln. Die erstgenannte Gruppe wird vermutlich von verschiedenen medialen Lernformen deutlich profitieren, etwa von Diskussionsforen. Für introspektive Lerner sind sie dagegen vermutlich eher eine Behinderung im Lernprozess. - Ein weiteres Beispiel: Es ist ebenfalls schon lange bekannt, dass verschiedene Lerner am besten lernen, indem sie beobachten (Bandura 1978). Offensichtlich werden Spiegelneuronen aktiv, wenn sie entsprechende Bemühungen anderer Lerner sehen, so dass ihre Selbstmotivation und letztlich ihr Lernerfolg steigt. Hier ist anzunehmen, dass auch mediale Beispiele positiv wirken können (etwa aus Fernsehsendungen). Umgekehrt müssen andere Lerner selbst Erfahrungen machen, um sie erfolgreich speichern und weiterverarbeiten zu können. Die vermittelte Kommunikation stellt für sie also eine Beschränkung dar. Allerdings könnte es sein, dass für Lerner, die individuelle Konzepte für ihr Lernen benötigen, einen Vorteil durch und in Online-Lernumgebungen haben - dort zumindest im Vergleich zum ,beobachtenden Lernertyp'. Wir haben hier also - zumindest theoretisch eine spezifische Zuordnung von Lernertyp und unterschiedlichen Szenarien medialen Lernens. Weiters benötigen unterschiedliche Lernertypen eine jeweils unterschiedlich dichte und umfangreiche Menge von Informationen, um letztlich von den Lehr- und Lernbemühungen profitieren zu können. Diese Informationen sind notwendig, um im Rahmen metakognitiver Vorgänge den Aufwand, die Probleme und den Nutzen des jeweiligen Lernprozesses abgewogen (Hayes, Allisnson 1998). Vielleicht erklärt dies zunächst die Befunde von Baruch und Nicholson (1997), die beobachtet haben, dass sich Lerner mit hoher Unsicherheitsvermeidungstendenz - die also eine nur 
geringe Bereitschaft haben, Risiken einzugehen und ohne direkte Sicherheit zu leben - mit Selbstlernmedien eher unwohl fühlen. Es gibt aber auch Paralellen zur weiteren oben beschrieben Beobachtung: Da Online-Medien den Grad an Mehrdeutigkeit sowohl bezüglich der Aufgaben, als auch der Erwartungen an den jeweiligen Lerner (und des Lerners an sich selbst) erhöhen, sind Lerner mit einer geringeren Toleranz bezüglich Unsicherheitsvermeidungen dort im Nachteil, während Lerner höheren Grad an Unsicherheitsvermeidung gar weitere Vorteile generieren können. Natürlich ist wünschenswert, $\mathrm{zu}$ wissen, ob und wie mediengestützte Lehrmaterialien sinnvoll und effizient eingesetzt werden können. Bei der Abschätzung, ob der Einsatz überhaupt sinnvoll ist, erscheint die bereits beschriebene Beobachtung bedeutsam, dass mediengestütztes Lernen grundsätzlich zeitintensiver als traditionelles Lernen ist. Demnach ist es sowohl für die Dozenten, als auch für die Lerner wichtig, mediengestützte Lernmaterialien so zu nutzen, dass der inhaltliche Mehrwert entsprechend groß ausfällt und die Mehrzahl der Lerner davon profitiert. Die Systematik müsste also (zumindest) folgende Ebenen berücksichtigen:

1. wie wirkt das Medium: Buch, Film (auf Computer, Fernsehgerät, Smartphone?), CD-Rom bzw. DVD, VoIP (z.B. Skype), Lernplattformen, Apps?

2. welche Inhalte: z.B. welche Sprache? Grammatik Landeskunde oder Ausspracheübungen? Grammatik für Muttersprachler, Erwerb der ersten Fremdsprache, plurilingualistischer Unterricht?

3. welcher Lerner: visueller, auditiver oder kinesthetischer Lernertyp? ein Kind oder ein erwachsener Lerner? ein ein-, zwei- oder gar mehrsprachiger Lerner?

Fraglich ist aber, ob sich eine Systematik entwickeln lässt, die Inhalte, Nutzertyp und Medium koppelt. Dies scheint mir jedoch, wie bereits angedeutet, kaum möglich zu sein, da bereits die genannten Variablen und ihre Wechselwirkungen $\mathrm{zu}$ viele unterschiedliche Situationen entstehen lassen. $\mathrm{Zu}$ den vielfältigen und großteils noch unerforschten Wechselwirkungen zwischen den beschriebenen drei Hauptvariablen kommen weitere und stets neue Variablen, nicht zuletzt die technische Entwicklung, die die Medien selbst immer weiter verändert und neue Situationen evoziert. Eine mögliche Reaktion ist aber, zumindest die Einsicht in die Wirkungsweise unterschiedlicher Variablen stets mitzuberücksichtigen. Dies ist weniger ,neu' (und weniger schwierig in der Umsetzung), als es klingt, denn in der Tat erfordert jeder Unterricht eine wechselseitige Anpassung des Lehrenden und des/der Lerner. Wichtig ist also, zunächst der Intuition des Lehrenden (und des/der Lernenden) zu vertrauen und nicht mediengestützte Lernprozesse zu fordern, wo sie kontraproduktiv oder auch nur problematisch in der Aufwand-Ergebnis-Relation wäre, nur weil Medien noch immer ,schick' und ,modern' sind. 


\section{LITERATURVERZEICHNIS}

Bailey, B.P., Iqbal, S.T., 2007. Understanding Changes in Mental Workload during Execution of Goal-directed Tasks and its Application for Interruption Management. In: ACM Transactions on Computer-Human Interaction. Vol 14, no. 21:1: 21-28.

Bandura, A., 1978. The Self-system in Reciprocal Determinism. In: American Psychologist. Vol. 33, 344-358.

Bangert, A.W., 2008. The Influence of Social Presence and Teaching Presence on the Quality of Online Critical Inquiry. In: Journal of Computing in Higher Education. Vol. 20, 34-61.

Baruch, Y., Nicholson, N., 1997. Home, Sweet Work: Requirements for Effective Homeworking. In: Journal of General Management. Vol. 23, no. 2, 15-30.

Bufe, W., Giessen, H.W. (Hrsg.), 2005. Videoconferencing grenzübergreifend - La visioconference transfrontaliere. Paris : L'Harmattan.

Clark, R.E., 1983. Reconsidering Research on Learning from Media. In: Review of Educational Research. Vol. 53, no. 4, 445-459.

Daft, R.L., Lengel, R.H., 1984. Information Richness: a New Approach to Managerial Behavior and Organizational Design. In: Cummings, L.L., Staw, B.M. (Hrsg.). Research in Organizational Behavior. Homewood, IL: JAI Press. Vol. 6, 191-233.

Ehlers, U.-D., 2004. Qualität im E-Learning aus Lernersicht. Grundlagen, Empirie und Modellkonzeption subjektiver Qualität. Wiesbaden: VS.

Ferrari, M., Sternberg, R.J., 1998. The Development of Mental Abilities and Styles. In: Damon, W., Kuhn, D., Siegler, R.S. (Hrsg.). Handbook of Child Psychology. Vol. 2. New York: Wiley, 899-946.

Flavell, J.H., 1992. Cognitive Development: Past, Present, and Future. In: Developmental Psychology. Vol. 28, 998-1005.

Fussell, S.R., Benimoff, N.I., 1995. Social and Cognitive Processes in Interpersonal Communication: Implications for Advanced Telecommunications Technologies. In: Human Factors. Vol. 37, 228-250.

Gardner, H., 2009. Fünf Kompetenzen für die Zukunft. In: Giessen, H. (Hrsg.). Medienpädagogik: Emotionale Intelligenz in der Schule. Unterrichten mit Geschichten. Weinheim-Basel: Beltz, 138-149.

Giessen, H.W., 2004. Sprachnetzwerke in Grenzräumen. Saarbrücken: LPM 2004.

Giessen, H.W., 2006. „Videosegmente als authentische Lehrmaterialien für den Sprachunterricht in computergestützten Multimedia-Produktionen?“. In: Beiträge zur Fremdsprachenvermittlung. Heft 45, 91-96. http://www.vep-landau.de/bzf/2006_45/07_Giessen_091096.pdf. (28.05.2013).

Grigorenko, E.L., Sternberg, R.J., 1995. Thinking styles. In: Saklogske, D.H. Zeidner, M. (eds). International Handbook of Personality and Intelligence. London: Plenum, 205-229.

Hayes, J., Allinson, C.W., 1998. Cognitive Style and the Theory and Practice of Individual and Collective Learning in Organizations. In: Human Relations. Vol. 51, 847-871.

Hazeltine, E., Ruthruff, E., Remington, R.W. 2006. The Role of Input and Output Modality Pairings in Dual-task Performance: Evidence for Content-dependent Central Interference. In: Cognitive Psychology. Vol. 52, 291-345.

Kariya, S., 2003. Online Education Expands and Evolves. In: IEEE Spectrum. Vol. 40, 49-51.

Lewin, K., 1942. Field Theory of Learning. In: Yearbook of the National Society for the Study of Education. Vol. 41, part 2, 215-242.

McLuhan, H.M., 1962. The Gutenberg Galaxy: The Making of Typographic Man. Toronto: University of Toronto Press. 
Mason, R., 2004. Online Education Using Learning Objects. In: British Journal of Educational Technology. Vol. 35, 752-754.

Montgomery, D.A., 1999. Human Sensitivity to Variability Information in Detection Decisions. In: Human Factors. Vol. 41, 90-105.

Pillay, H., 1998. An Investigation of the Effect of Individual Cognitive Preferences on Learning Through Computer-based Instruction. In: Educational Psychology. Vol. 18, 171-182.

Pitman, A.J., Gosper, M., Rich, D.C., 1999. Internet-Based Teaching in Geography at Macquarie University: An Analysis of Student Use. In: Australian Journal of Educational Technology. Vol. 15, 167-187.

Rehder B., Hoffman A.B., 2005. Eye tracking and Selective Attention in Category Learning. In: Cognitive Psychology. Vol. 51,1-41.

Schulman, A.H., Sims, R.L., 1999. Learning in an Online Format versus an In-class Format: An Experimental Study. In: Technological Horizons in Education - T.H.E. Journal. Vol. 26, no. 11, $59-65$.

Scott, D., Durnell-Cramton, C., Gauvin, S., Steinke, G., Patterson, K., 1997. Internet-based Collaborative Learning. An Empirical Evaluation. In: Proceedings 3rd Ausweb Conference, Lismore, Australia, 1997. http://ausweb.scu.edu.au/proceedings/donscott/index.html; 4. November 2011.

Sternberg, R.J., 1997. Thinking Styles. New York: Cambridge University Press.

Sternberg, R.J., Grigorenko, E.L., 1997. Are Cognitive Styles Still in Style. In: American Psychologist. Vol. 52, 700-712.

Tamim, R.M., Bernard, R.M., Borokhovski, E., Abrami, P.C., Schmid, R.F. 2011. What Forty Years of Research Says About the Impact of Technology on Learning: A Second-Order Meta-Analysis and Validation Study. In: Review of Educational Research. March 2011, Vol. 81, no. 1, 4-28.

Turkle, S. 1984. The Second Self: Computers and the Human Spirit, Cambridge, Mass.: MIT-Press.

Usip, E.E., Bee, R.H., 1998. Economics: A Discriminant Analysis of Student's Perceptions of Web-Based Learning. In: Social Science Computer Review. Vol. 16, 16-29.

Wallace, D.R., Mutooni, P., 1997. A Comparative Evaluation of World Wide Web-based and Classroom Teaching. In: Journal of Engineering Education. Vol. 86, 211-219.

Ward, M., Newlands, D., 1998. Use of the Web in Undergraduate Teaching. In: Computers and Education. Vol. 31, 171-184.

Workman, M., 2004. Performance in Computer-based and Computer-aided Education: Do Cognitive Styles Make a Difference?, In: Journal of Computers in Human Behavior. Vol. 20, 517-534. 